\title{
Correction
}

\section{Correction to: Quantum-tunneling transitions and exact statistical mechanics of bistable systems with parametrized Dikandé-Kofané double-well potentials}

F. Naha Nzoupe ${ }^{1, a}$, Alain M. Dikandé ${ }^{2,1, b} \mathbb{O}$, and S. E. Mkam Tchouobiap ${ }^{1, c}$

${ }^{1}$ Laboratory of Research on Advanced Materials and Nonlinear Science (LaRAMaNS), Department of Physics, Faculty of Science, University of Buea, PO Box 63, Buea, Cameroon

2 Present Address: Max-Planck Institute for the Physics of Complex Systems, Nöthnitzer Strasse 38, 01187 Dresden, Germany

Published online 7 February 2022

(C) EDP Sciences, SIF and Springer-Verlag GmbH Germany, part of Springer Nature 2022

\section{Correction to:}

Eur. Phys. J. B (2022) 95

https://doi.org/10.1140/epjb/s10051021-00266-w

The following affiliation has been added:
Max-Planck Institute for the Physics of Complex Systems, Nöthnitzer Strasse 38, 01187 Dresden, Germany

The original article has been corrected.

The original article can be found online at https://doi.org/ 10.1140/epjb/s10051-021-00266-w.

\footnotetext{
${ }^{\mathrm{a}}$ e-mail: fernand.naha.nz@gmail.com

b e-mail: dikande.alain@ubuea.cm (corresponding author)

c e-mail: esmkam@yahoo.com
} 